\title{
Inhibition of DNA ejection from bacteriophage by $\mathrm{Mg}^{+2}$ counterions
}

\author{
Sell Lee, ${ }^{1}$ C. V. Tran, ${ }^{2}$ and T. T. Nguyen ${ }^{11}$ \\ ${ }^{1}$ School of Physics, Georgia Institute of Technology, 837 State Street, Atlanta, \\ Georgia 30332-0430 \\ ${ }^{2}$ School of Chemistry and Biochemistry, Georgia Institute of Technology, \\ 901 Atlantic Drive, Atlanta, Georgia 30332-0400
}

(Dated: 2 November 2018)

The problem of inhibiting viral DNA ejection from bacteriophages by multivalent counterions, specifically $\mathrm{Mg}^{+2}$ counterions, is studied. Experimentally, it is known that $\mathrm{MgSO}_{4}$ salt has a strong and non-monotonic effect on the amount of DNA ejected. There exists an optimal concentration at which the minimum amount of DNA is ejected from the virus. At lower or higher concentrations, more DNA is ejected from the capsid. We propose that this phenomenon is the result of DNA

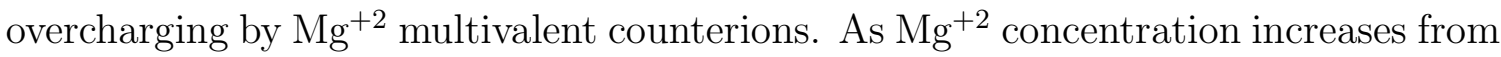
zero, the net charge of DNA changes from negative to positive. The optimal inhibition corresponds to the $\mathrm{Mg}^{+2}$ concentration where DNA is neutral. At lower/higher concentrations, DNA genome is charged. It prefers to be in solution to lower its electrostatic self-energy, which consequently leads to an increase in DNA ejection. By fitting our theory to available experimental data, the strength of DNA-DNA short range attraction energies, mediated by $\mathrm{Mg}^{+2}$, is found to be $-0.004 k_{B} T$ per nucleotide base. This and other fitted parameters agree well with known values from other experiments and computer simulations. The parameters are also in aggreement qualitatively with values for tri- and tetra-valent counterions.

PACS numbers: 81.16.Dn, 87.16.A-, 87.19.rm 


\section{INTRODUCTION}

Most bacteriophages, or viruses that infect bacteria, are composed of a DNA genome coiling inside a rigid, protective capsid. It is well-known that the persistence length, $l_{p}$, of DNA is about $50 \mathrm{~nm}$, comparable to or larger than the inner diameter of the viral capsid. The genome of a typical bacteriophage is about 10 microns or 200 persistence lengths. Thus the DNA molecule is considerably bent and strongly confined inside the viral capsid resulting in a substantially pressurized capsid with internal pressure as high as 50 atm [1 4]. It has been suggested that this pressure is the main driving force for the ejection of the viral genome into the host cell when the capsid tail binds to the receptor in the cell membrane, and subsequently opens the capsid. This idea is supported by various experiments both in vivo and in vitro [2, 3, 5-10]. The in vitro experiments additionally revealed possibilities of controlling the ejection of DNA from bacteriophages. One example is the addition of PEG (polyethyleneglycol), a large molecule incapable of penetrating the viral capsid. A finite PEG concentration in solution produces an apparent osmotic pressure on the capsid. This in turn leads to a reduction or even complete inhibition of the ejection of DNA.

Since DNA is a strongly charged molecule in aqueous solution, the screening condition of the solution also affects the ejection process. At a given external osmotic pressure, by varying the salinity of solution, one can also vary the amount of DNA ejected. Interestingly, it has been shown that monovalent counterions such as $\mathrm{NaCl}$ have a negligible effect on the DNA ejection process [2]. In contrast, multivalent counterions such as $\mathrm{Mg}^{+2}, \mathrm{CoHex}^{+3}$ (Co-hexamine), $\mathrm{Spd}^{+3}$ (spermidine) or $\mathrm{Spm}^{+4}$ (spermine) exert strong effect. In this paper, we focus on the role of $\mathrm{Mg}^{+2}$ divalent counterion on DNA ejection. In Fig. 1, the percentage of ejected DNA from bacteriophage $\lambda$ (at 3.5 atm external osmotic pressure) from the experiment of Ref. 10 and 11 are plotted as a function of $\mathrm{MgSO}_{4}$ concentration (solid circles). The three colors correspond to three different sets of data. Evidently, the effect of multivalent counterions on the DNA ejection is non-monotonic. There is an optimal $\mathrm{Mg}^{+2}$ concentration where the minimum amount of DNA genome is ejected from the phages.

The general problem of understanding DNA condensation and interaction in the presence of multivalent counterions is rather complex, as evident by the large literature dedicated to this subject. This is especially true in the case of divalent counterions because many physical factors involved are energetically comparable to each other. Most studies related to 


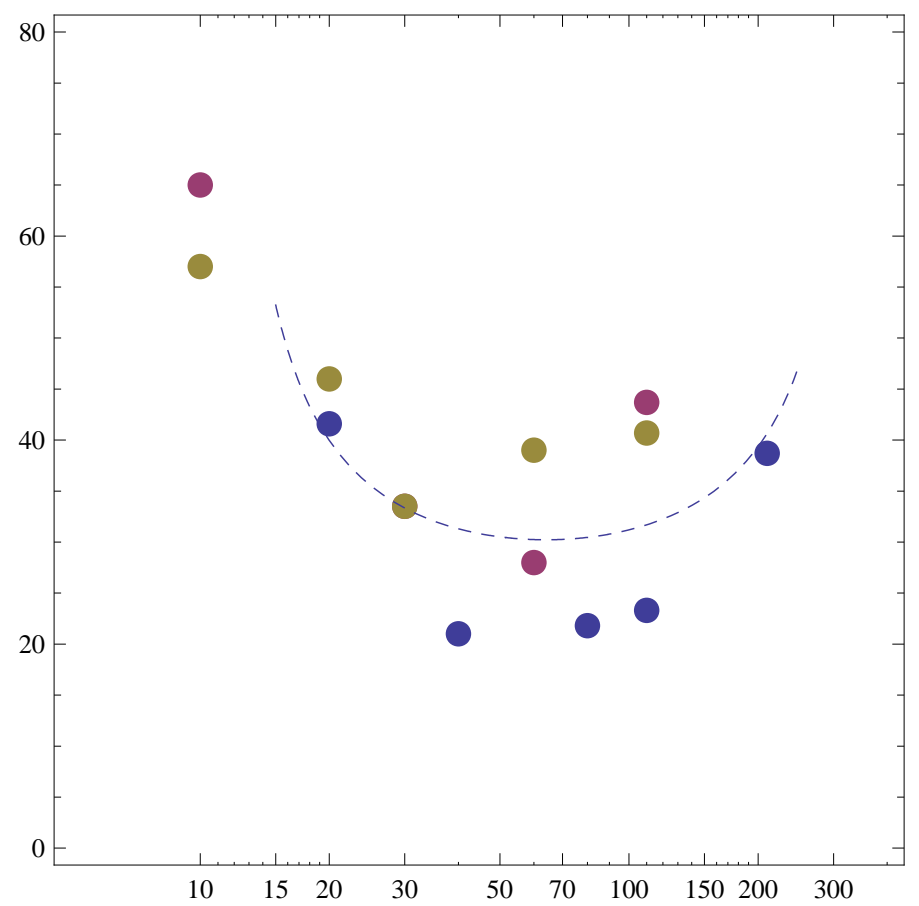

FIG. 1. (Color online) Inhibition of DNA ejection depends on $\mathrm{MgSO}_{4}$ concentration for bacteriophage $\lambda$ at 3.5 atm external osmotic pressure. Solid circles represent experimental data from Ref. 10 and 11], where different colors corresponds to different experimental batch. The dashed line is a theoretical fit of our theory. See Sec. IV

DNA screening in the presence of divalent counterions have focused on ion specific effects. For example, in Ref. 10, hydration effects were proposed to explain the data of DNA ejection in the presence of $\mathrm{MgCl}_{2}$ salt where the minimum has not yet been observed for salt concentration upto $100 \mathrm{mM}$. In this paper, we focus on understanding the non-specific electrostatic interactions involved in the inhibition of DNA ejection by divalent counterions. We show that some aspects of the DNA ejection experiments can be explained within this framework. Specifically, we propose that the non-monotonic behavior observed in Fig. 11 has similar physical origin to that of the phenomenon of the reentrant condensation of macroions in the presence of multivalent counterions. It is the result of $\mathrm{Mg}^{+2}$ ions inducing an effective attraction between DNA segments inside the capsid, and the so-called overcharging of DNA by multivalent counterions in free solution.

Specifically, the electrostatics of $\mathrm{Mg}^{+2}$ modulated DNA ejection from bacteriophages is following. Due to strong electrostatic interaction between DNA and $\mathrm{Mg}^{+2}$ counterions, the 
counterions condense on the DNA molecule. As a result, a DNA molecule behaves electrostatically as a charged polymer with the effective net charge, $\eta^{*}$ per unit length, equal to the sum of the "bare" DNA charges, $\eta_{0}=-1 e / 1.7 \AA$, and the charges of condensed counterions. There are strong correlations between the condensed counterions at the DNA surface which cannot be described using the standard Poisson-Boltzmann mean-field theory. Strongly correlated counterion theories, various experiments and simulations [12 16] have showed that when these strong correlations are taken into account, $\eta^{*}$ is not only smaller than $\eta_{0}$ in magnitude but can even have opposite sign: this is known as the charge inversion phenomenon. The degree of counterion condensation, and correspoly the value of $\eta^{*}$, depends logarithmically on the concentration of multivalent counterions, $N_{Z}$. As $N_{Z}$ increases from zero, $\eta^{*}$ becomes less negative, neutral and eventually positive. We propose that the multivalent counterion concentration, $N_{Z, 0}$, where DNA's net charge is neutral corresponds to the optimal inhibition due to $\mathrm{Mg}^{+2}$-induced DNA-DNA attraction inside the capsid. At counterion concentration $N_{Z}$ lower or higher than $N_{Z, 0}, \eta^{*}$ is either negative or positive. As a charged molecule at these concentrations, DNA prefers to be in solution to lower its electrostatic self-energy (due to the geometry involved, the capacitance of DNA molecule is higher in free solution than in the bundle inside the capsid). Accordingly, this leads to a higher percentage of ejected viral genome.

The fact that $\mathrm{Mg}^{+2}$ counterions can have such strong influence on DNA ejection is highly non-trivial. It is well-known that $\mathrm{Mg}^{+2}$ ions do not condense or only condense partially free DNA molecules in aqueous solution [17, 18]. Yet, they exert strong effects on DNA ejection from bacteriophages. We argue that this is due to the entropic confinement of the viral capsid. Unlike free DNA molecules in solution, DNA packaged inside capsid are strongly bent and the thermal fluctuations of DNA molecule is strongly suppressed. It is due to this unique setup of the bacteriophage where DNA is pre-packaged by a motor protein during virus assembly that $\mathrm{Mg}^{+2}$ ions can induce attractions between DNA. It should be mentioned that $\mathrm{Mg}^{+2}$ counterions have been shown experimentally to condense DNA in another confined system: the DNA condensation in two dimension [19]. Recent computer simulations [20, 21] also show that if the lateral motion of DNA is restricted, divalent counterions can induced DNA condensation. The strength of DNA-DNA attraction energy mediated by divalent counterions is comparable to the results presented in this paper. These facts strongly support our proposed argument. 
The dashed line in Fig. 1 is a fit of our theoretical result to the experimental data for $\mathrm{MgSO}_{4}$. The optimal $\mathrm{Mg}^{+2}$ concentration is shown to be $N_{Z, 0}=64 \mathrm{mM}$. The $\mathrm{Mg}^{+2}$-mediated attraction between DNA double helices is found to be $-0.004 k_{B} T /$ base ( $k_{B}$ is the Boltzmann constant and $T$ is the temperature of the system). As discussed later in Sec. IV, these values agree well with various known parameters of other DNA systems.

The organization of the paper as follows; In Sec. III, a brief review of the phenomenon of overcharging DNA by multivalent counterions is presented. In Sec. III, the semi-empirically theory is fit to the experimental data of DNA ejection from bacteriophages. In Sec. IV, the obtained fitting parameters is discussed in the context of various other experimental and simulation studies of DNA condensation by divalent counterions. Finally, we conclude our paper in Sec. V.

\section{OVERCHARGING OF DNA BY MULTIVALENT COUNTERIONS}

In this section, let us briefly visit the phenomenal of overcharging of DNA by multivalent counterions to introduce various physical parameters involved in our theory. Standard linearized mean field theories of electrolyte solution states that in solutions with mobile ions, the Coulomb potential of a point charge, $q$, is screened exponentially beyond a Debye-Hückel $(\mathrm{DH})$ screening radius, $r_{s}$ :

$$
V_{D H}(r)=\frac{q}{r} \exp \left(-r / r_{s}\right) .
$$

The DH screening radius $r_{s}$ depends on the concentrations of mobile ions in solution and is given by:

$$
r_{s}=\sqrt{\frac{D k_{B} T}{4 \pi e^{2} \sum_{i} c_{i} z_{i}^{2}}}
$$

where $c_{i}$ and $z_{i}$ are the concentration and the valence of mobile ions of species $i, e$ is the charge of a proton, and $D \approx 78$ is the dielectric constant of water.

Because DNA is a strongly charged molecule in solution, linear approximation breaks down near the DNA surface because the potential energy, $e V_{D H}(r)$, would be greater than $k_{B} T$ in this region. It has been shown that, within the general non-linear meanfield PoissonBoltzmann theory, the counterions would condense on the DNA surface to reduce its surface potential to be about $k_{B} T$. This so-called Manning counterion condensation effect leads to 
an "effective" DNA linear charge density:

$$
\eta_{c}=-D k_{B} T / e
$$

In these mean field theories, the charge of a DNA remains negative at all ranges of ionic strength of the solution. The situation is completely different when DNA is screened by multivalent counterions such as $\mathrm{Mg}^{2+}, \mathrm{Spd}^{3+}$ or $\mathrm{Spm}^{4+}$. These counterions also condense on DNA surface due to theirs strong attraction to DNA negative surface charges. However, unlike their monovalent counterparts, the electrostatic interactions among condensed counterions are very strong due to their high valency. These interactions are even stronger than $k_{B} T$ and mean field approximation is no longer valid in this case. Counterintuitive phenomena emerge when DNA molecules are screened by multivalent counterions. For example, beyond a threshold counterion concentration, the multivalent counterions can even over-condense on a DNA molecule making its net charge positive. Furthermore, near the threshold concentration, DNA molecules are neutral and they can attract each other causing condensation of DNA into macroscopic bundles (the so-called like-charged attraction phenomenon).

To understand how multivalent counterions overcharge DNA molecules, let us write down the balance of the electro-chemical potentials of a counterion at the DNA surface and in the bulk solution.

$$
\mu_{c o r}+Z e \phi(a)+k_{B} T \ln \left[N_{Z}(a) v_{o}\right]=k_{B} T \ln \left[N_{Z} v_{o}\right]
$$

Here $v_{o}$ is the molecular volume of the counterion, $Z$ is the counterion valency. $\phi(a)$ is the electrostatic surface potential at the dressed DNA. Approximating the dressed DNA as a uniformly charged cylinder with linear charged density $\eta^{*}$ and radius $a, \phi(a)$ can be written as:

$$
\phi(a)=\frac{2 \eta^{*}}{D} \frac{K_{0}\left(a / r_{s}\right)}{\left(a / r_{s}\right) K_{1}\left(a / r_{s}\right)} \simeq \frac{2 \eta^{*}}{D} \ln \left(1+\frac{r_{s}}{a}\right)
$$

where $K_{0}$ and $K_{1}$ are Bessel functions (this expression is twice the value given in Ref. 22 because we assume that the screening ion atmosphere does not penetrate the DNA cylinder). In Eq. (41), $N_{Z}(a)$ is the local concentration of the counterion at the DNA surface:

$$
N_{Z}(a) \approx \sigma_{0} /(Z e \lambda)=\eta_{0} /(2 \pi a Z e \lambda)
$$

where $\sigma_{0}=\eta_{0} / 2 \pi a$ is the bare surface charge density of a DNA molecule and the GouyChapman length $\lambda=D k_{B} T / 2 \pi \sigma_{0} Z e$ is the distance at which the potential energy of a 
counterion due to the DNA bare surface charge is one thermal energy $k_{B} T$. The term $\mu_{\text {cor }}$ in Eq. (4) is due to the correlation energies of the counterions at the DNA surface. It is this term which is neglected in mean-field theories. Several approximate, complementary theories, such as strongly correlated liquid [12, 13, and 23], strong coupling [14, 16] or counterion release [24, 25] have been proposed to calculate this term. Although with varying degree of analytical complexity, they have similar physical origins. In this paper, we followed the theory presented in Ref. 13. In this theory, the strongly interacting counterions in the condensed layer are assumed to form a two-dimensional strongly correlated liquid on the surface of the DNA (see Fig. 2). In the limit of very strong correlation, the liquid form a two-dimensional Wigner crystal (with lattice constant $A$ ) and $\mu_{\text {cor }}$ is proportional to the interaction energy of the counterion with background charges of its Wigner-Seitz cell. Exact calculation of this limit gives [13]:

$$
\mu_{\text {cor }} \approx-1.65 \frac{(Z e)^{2}}{D r_{W S}}=-1.17 \frac{1}{D}(Z e)^{3 / 2}\left(\frac{\eta_{0}}{a}\right)^{1 / 2}
$$

Here $r_{W S}=\sqrt{\sqrt{3} A^{2} / 2 \pi}$ is the radius of a disc with the same area as that of a Wigner-Seitz cell of the Wigner crystal (see. Fig. 2). It is easy to show that for multivalent counterions, the so-called Coulomb coupling (or plasma) parameter, $\Gamma=(Z e)^{2} / D r_{W S} k_{B} T$, is greater than one. Therefore, $\left|\mu_{\text {cor }}\right|>k_{B} T$, and thus cannot be neglected in the balance of chemical potential, Eq. (4).

Knowing $\mu_{\text {cor }}$, one can easily solve Eq. (4) to obtain the net charge of a DNA for a given counterion concentration:

$$
\eta^{*}=-\frac{D k_{B} T}{2 Z e} \frac{\ln \left(N_{Z, 0} / N_{Z}\right)}{\ln \left(1+r_{s} / a\right)},
$$

where the concentration $N_{Z, 0}$ is given by:

$$
N_{Z, 0}=N_{Z}(a) e^{-\left|\mu_{c o r}\right| / k_{B} T}
$$

Eq. (8) clearly shows that for counterion concentrations higher than $N_{Z, 0}$, the DNA net charge $\eta^{*}$ is positive, indicating the over-condensation of the counterions on DNA. In other words, DNA is overcharged by multivalent counterions at these concentrations. Notice Eq. (7) shows that, for multivalent counterions $Z \gg 1, \mu_{\text {cor }}$ is strongly negative for multivalent counterions, $\left|\mu_{\text {cor }}\right| \gg k_{B} T$. Therefore, $N_{Z, 0}$ is exponentially smaller than $N_{Z}(a)$ and a realistic concentration obtainable in experiments. 


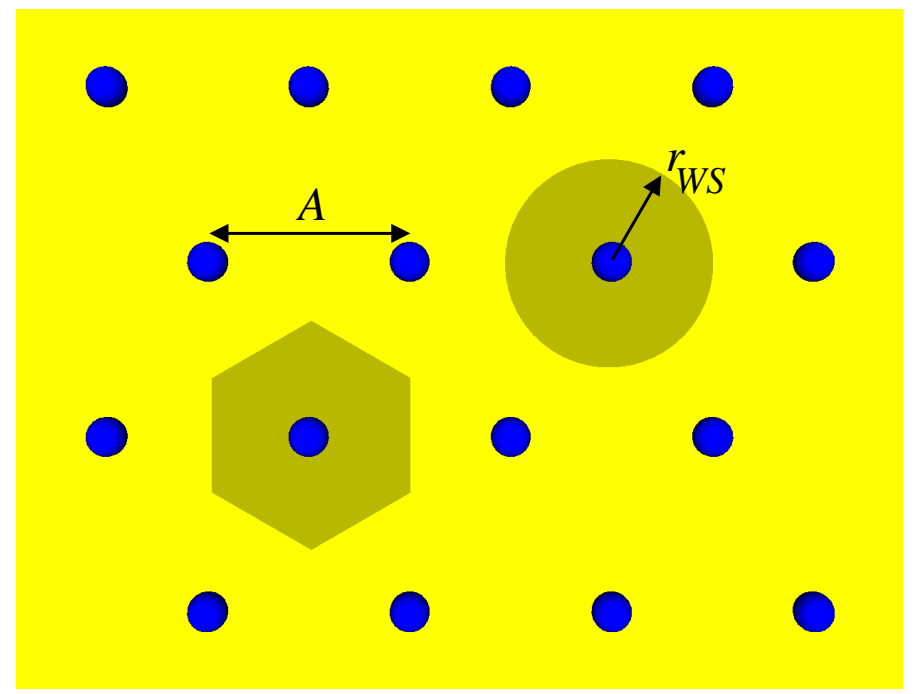

FIG. 2. (Color online) Strong electrostatic interactions among condensed counterions lead to the formation of a strongly correlated liquid on the surface of the DNA molecule. In the limit of very strong interaction, this liquid forms a two-dimensional Wigner crystal with lattice constant $A$. The shaded hexagon is a Wigner-Seitz cell of the background charge. It can be approximated as a disc of radius $r_{W S}$.

Besides the overcharging phenomenon, DNA molecules screened by multivalent counterions also experience the counterintuitive like-charge attraction effect. This short range attraction between DNA molecules can also be explained within the framework of the strong correlated liquid theory. Indeed, in the area where DNA molecules touch each other, each counterion charge is compensated by the "bare" background charge of two DNA molecules instead of one (see Fig. 3). Due to this doubling of background charge, each counterion condensed in this region gains an energy of:

$$
\delta \mu_{\text {cor }} \approx \mu_{\text {cor }}\left(2 \eta_{0}\right)-\mu_{\text {cor }}\left(\eta_{0}\right) \simeq-0.46 \frac{1}{D}(Z e)^{3 / 2}\left(\frac{\eta_{0}}{a}\right)^{1 / 2}
$$

As a result, DNA molecules experience a short range correlation-induced attraction. Approximating the width of this region to be on the order of the Wigner crystal lattice constant $A$, the DNA-DNA attraction per unit length can be calculated:

$$
\mu_{\mathrm{DNA}} \simeq-\frac{2 \sqrt{2 a A} \sigma_{0}}{Z e}\left|\delta \mu_{\text {cor }}\right| \simeq-0.34 \frac{1}{D} \eta_{0}^{5 / 4}\left(\frac{Z e}{a}\right)^{3 / 4}
$$

The combination of the overcharging of DNA molecules and the like charged attraction phenomena (both induced by multivalent counterions) leads to the so-called reentrant 


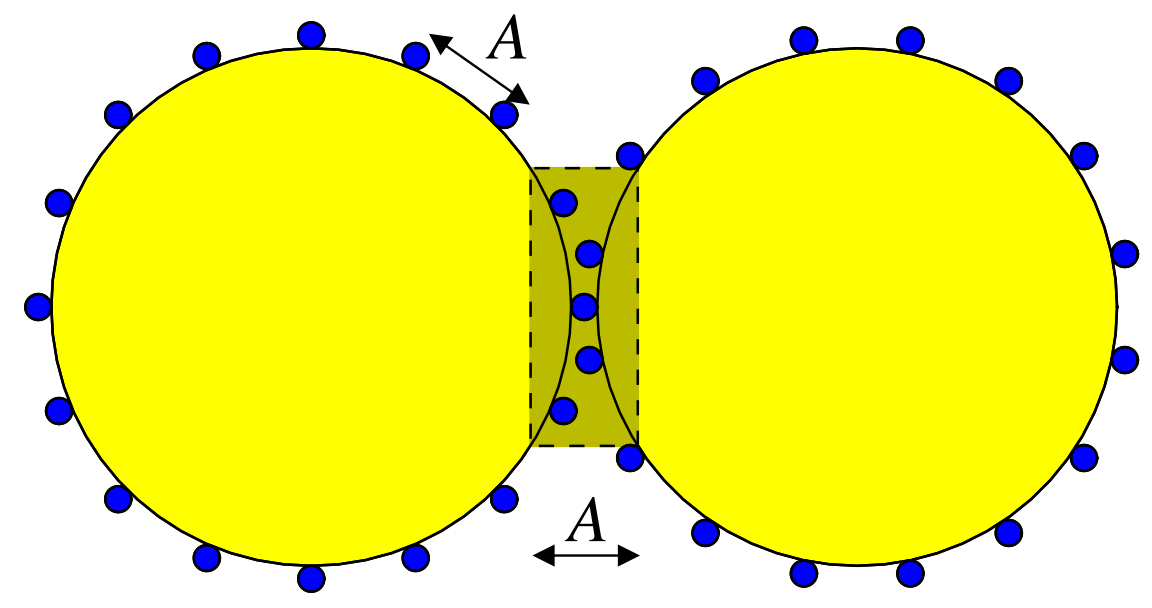

FIG. 3. (Color online) Cross section of two touching DNA molecules (large yellow circles) with condensed counterions (blue circles). At the place where DNA touches each other (the shaded region of width $A$ shown), the density of the condensed counterion layer doubles and additional correlation energy is gained. This leads to a short range attraction between the DNA molecules.

condensation of DNA. At small counterion concentrations, $N_{Z}$, DNA molecules are undercharged. At high counterion concentrations, $N_{Z}$, DNA molecules are overcharged. The Coulomb repulsion between charged DNA molecules keeps individual DNA molecules apart in solution. At an intermediate range of $N_{Z}$, DNA molecules are mostly neutral. The short range attraction forces are able to overcome weak Coulomb repulsion leading to their condensation. In this paper, we proposed that this reentrant behavior of DNA condensation as function of counterion concentration is the main physical mechanism behind the non-monotonic dependence of DNA ejection from bacteriophages as a function of the $\mathrm{Mg}^{+2}$ concentrations.

\section{THEORETICAL CALCULATION OF DNA EJECTION FROM BACTERIOPHAGE}

We are now in the position to obtain a theoretical description of the problem of DNA ejection from bacteriophages in the presence of multivalent counterions. We begin by writing the total energy of a viral DNA molecule as the sum of the energy of DNA segments ejected 
outside the capsid with length $L_{o}$ and the energy of DNA segments remaining inside the capsid with length $L_{i}=L-L_{o}$, where $L$ is the total length of the viral DNA genome:

$$
E_{\text {tot }}\left(L_{o}\right)=E_{\text {in }}\left(L_{i}\right)+E_{\text {out }}\left(L_{o}\right)
$$

Because the ejected DNA segment is under no entropic confinement, we neglect contributions from bending energy and approximate $E_{\text {out }}$ by the electrostatic energy of a free DNA of the same length in solution:

$$
E_{\text {out }}\left(L_{o}\right)=-L_{o}\left(\eta^{* 2} / D\right) \ln \left(1+r_{s} / a\right),
$$

where the DNA net charge, $\eta^{*}$, for a given counterion concentration is given by Eq. (88). The negative sign in Eq. (13) signifies the fact that the system of the combined DNA and the condensed counterions is equivalent to a cylindrical capacitor under constant charging potential. As shown in previous section, we expect the $\eta^{*}$ to be a function of the multivalent counterion concentration $N_{Z}$ and can be positive when $N_{Z}>N_{Z, 0}$. In the limit of strongly correlated liquid, $N_{Z, 0}$ is given in Eq. (9). However, the exponential factor in this equation shows that an accurate evaluation of $N_{Z, 0}$ is very sensitive to an accurate calculation of the correlation chemical potential $\mu_{c o r}$. For practical purposes, the accurate calculation of $\mu_{c o r}$ is a highly non-trivial task. One would need to go beyond the flat two-dimensional Wigner crystal approximation and takes into account not only the non-zero thickness of the condensed counterion layer but also the complexity of DNA geometry. Therefore, within the scope of this paper, we are going to consider $N_{Z, 0}$ as a phenomenological constant concentration whose value is obtained by fitting the result of our theory to the experimental data.

The energy of the DNA segment inside the viral capsid comes from the bending energy of the DNA coil and the interaction between neighboring DNA double helices:

$$
E_{\text {in }}\left(L_{i}, d\right)=E_{\text {bend }}\left(L_{i}, d\right)+E_{\text {int }}\left(L_{i}, d\right) .
$$

where $d$ is the average DNA-DNA interaxial distance.

There exists different models to calculate the bending energy of a packaged DNA molecules in literature [4, 8, 26 28]. In this paper, for simplicity, we employ the viral DNA packaging model used previously in Ref. 8, 26, 27. In this model, the DNA viral genome are assumed to simply coil co-axially inward with the neighboring DNA helices 


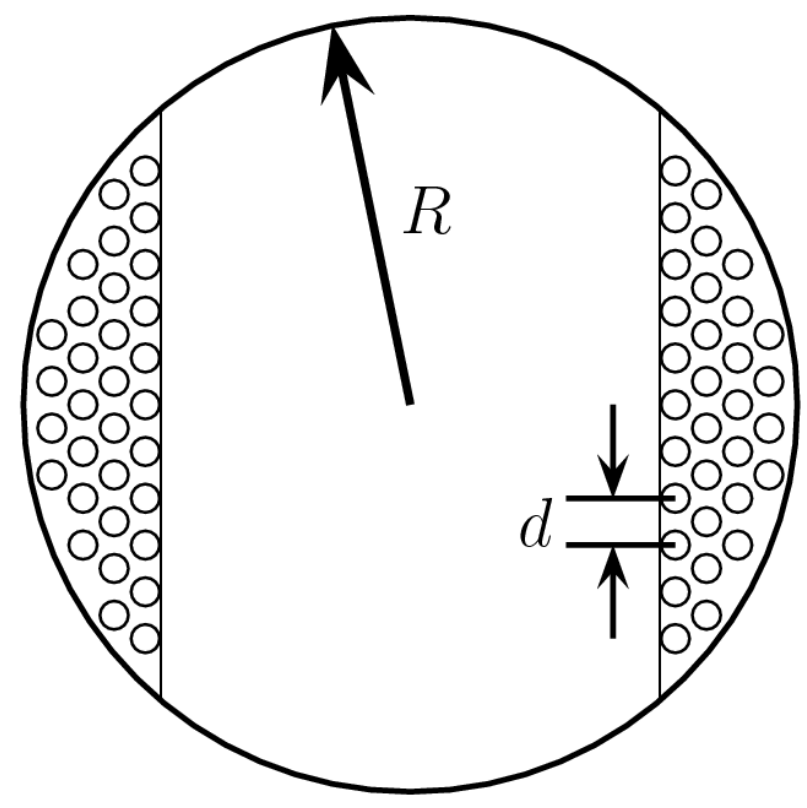

FIG. 4. A model of bacteriophage genome packaging. The viral capsid is modeled as a rigid spherical cavity. The DNA inside coils co-axially inward. Neighboring DNA helices form a hexagonal lattice with lattice constant $d$. A sketch for a cross section of the viral capsid is shown.

forming a hexagonal lattice with lattice constant $d$ (Fig. 4). For a spherical capsid, this model gives:

$$
E_{\text {bend }}\left(L_{i}, d\right)=\frac{4 \pi l_{p} k_{B} T}{\sqrt{3} d^{2}}\left\{-\left(\frac{3 \sqrt{3} L_{i} d^{2}}{8 \pi}\right)^{1 / 3}+R \ln \frac{R+\left(3 \sqrt{3} L_{i} d^{2} / 8 \pi\right)^{1 / 3}}{\left[\left(R^{2}-\left(3 \sqrt{3} L_{i} d^{2} / 8 \pi\right)^{2 / 3}\right]^{1 / 2}\right.}\right\},
$$

where $R$ is the radius of the inner surface of the viral capsid.

To calculate the interaction energy between neighboring DNA segments inside the capsid, $E_{\text {int }}\left(L_{i}, d\right)$, we assume that DNA molecules are almost neutralized by the counterions (the net charge, $\eta^{*}$ of the DNA segment inside the capsid is much smaller than that of the ejected segment because the latter has higher capacitance). In the previous section, we have shown that for almost neutral DNA, their interaction is dominated by short range attraction forces. Hence, one can approximate:

$$
E_{\text {int }}\left(L_{i}, d_{0}\right)=-L_{i}\left|\mu_{D N A}\right|
$$

Here, $d_{0}$ is the equilibrium interaxial distance of DNA bundle condensed by multivalent counterions. Due to the strongly pressurized viral capsid, the actual interaxial distance, $d$, between neighboring DNA double helices inside the capsid is smaller than the equilibrium 
distance, $d_{0}$, inside the condensate. The experiments from Ref. 17 provided an empirical formula that relates the restoring force to the difference $d_{0}-d$. Integrating this restoring force with $d$, one obtains an expression for the interaction energy between DNA helices for a given interaxial distance $d$ :

$$
E_{\text {int }}\left(L_{i}, d\right)=L_{i} \sqrt{3} F_{0}\left[\left(c^{2}+c d\right) \exp \left(\frac{d_{0}-d}{c}\right)-\left(c^{2}+c d_{0}\right)-\frac{1}{2}\left(d_{0}^{2}-d^{2}\right)\right]-L_{i}\left|\mu_{D N A}\right|,
$$

where the empirical values of the constants $F_{0}$ and $c$ are $0.5 \mathrm{pN} / \mathrm{nm}^{2}$ and $0.14 \mathrm{~nm}$ respectively.

As we shown in the previous section, like the parameter $N_{Z, 0}$, accurate calculation of $\mu_{D N A}$ is also very sensitive to an accurate determination of the counterion correlation energy, $\mu_{\text {cor }}$. Adopting the same point of view, instead of using the analytical approximation Eq. (11), we treat $\mu_{D N A}$ and $d_{0}$ as additional fitting parameters. In total, our semi-empirical theory has three fitting parameters $\left(N_{Z, 0}, \mu_{D N A}, d_{0}\right)$. From experimental data, we have three fitting constrains (the two coordinates of the minimum and the curvature of the curve $L_{o}\left(N_{Z}\right)$ in Fig. 1). Thus the theory does not contain unnecessary degrees of freedom.

\section{FITTING OF EXPERIMENT OF DNA EJECTION FROM BACTERIOPHAGES AND DISCUSSION}

Equation (12) together with equations (13), (14), (15) and (17) provide the complete expression for the total energy of the DNA genome of our semi-empirical theory. For a given external osmotic pressure, $\Pi_{o s m}$, and a given multivalent counterion concentration, $N$, the equilibrium value for the ejected DNA genome length, $L_{o}^{*}$, is the length that minimizes the total free energy $G\left(L_{o}\right)$ of the system, where

$$
G\left(L_{o}\right)=E_{t o t}\left(L_{o}\right)+\Pi_{o s m} L_{o} \pi a^{2}
$$

Here, $L_{o} \pi a^{2}$ is the volume of ejected DNA segments in aqueous solution. The specific fitting procedure is following. The energy $E_{i n}\left(L-L_{o}, d\right)$ of the DNA segment inside the capsid is minimized with respect to $d$ to acquire the optimal DNA-DNA interaxial distance for a given DNA ejected length, $d^{*}\left(L_{o}\right)$. Then, we substitute $E_{\text {tot }}\left(L_{o}\right)=E_{\text {in }}\left[L-L_{o}, d^{*}\left(L_{o}\right)\right]+E_{\text {out }}\left(L_{o}\right)$ into Eq. (18) and optimize $G\left(L_{o}\right)$ with respect to $L_{o}$ to obtain the equilibrium ejected length $L_{o}^{*}\left(\Pi_{o s m}, N\right)$. By fitting $L_{o}^{*}$ with experiment data we can obtain the values for the neutralizing counterion concentration, $N_{Z, 0}$, the $\mathrm{Mg}^{+2}-$ mediated DNA-DNA attraction, 
$-\left|\mu_{D N A}\right|$, and the equilibrium DNA-DNA distance $d_{0}$. The result of fitting our theoretical ejected length $L_{o}^{*}$ to the experimental data of Ref. 10 is shown in Fig. 1. In the experiment, wild type bacteriophages $\lambda$ was used, so $R=29 \mathrm{~nm}$ and $L=16.49 \mu \mathrm{m}$ [29]. $\Pi_{o s m}$ is held fixed at $3.5 \mathrm{~atm}$ and the $\mathrm{Mg}^{+2}$ counterion concentration is varied from $10 \mathrm{mM}$ to $200 \mathrm{mM}$. The fitted values are found to be $N_{Z, 0}=64 \mathrm{mM}, \mu_{D N A}=-0.004 k_{B} T$ per nucleotide base, and $d_{0}=2.73 \mathrm{~nm}$.

The strong influence of multivalent counterions on the process of DNA ejection from bacteriophage appears in several aspects of our theory and is easily seen by setting $d=d_{0}$, thus neglecting the weak dependence of $d$ on $L_{i}$ and using Eq. (16) for DNA-DNA interactions inside the capsid. Firstly, the attraction strength $\left|\mu_{D N A}\right|$ appears in the expression for the free energy, Eq. (18), with the same sign as $\Pi_{o s m}$ (recall that $L_{i}=L-L_{o}$ ). In other words, the attraction between DNA strands inside capsid acts as an additional "effective" osmotic pressure preventing the ejection of DNA from bacteriophage. This switch from repulsive DNA-DNA interactions for monovalent counterion to attractive DNA-DNA interactions for $\mathrm{Mg}^{+2}$ leads to an experimentally observed decrease in the percentage of DNA ejected from $50 \%$ for monovalent counterions to $20 \%$ for $\mathrm{Mg}^{+2}$ counterions at optimal inhibition $\left(N=N_{Z, 0}\right)$. Secondly, the electrostatic energy of the ejected DNA segment given by Eq. (13) is logarithmically symmetrical around the neutralizing concentration $N_{Z, 0}$. This is well demonstrated in Fig. [1 where the log-linear scale is used. This symmetry is also similar to the behavior of another system which exhibits charge inversion phenomenon, the non-monotonic swelling of macroion by multivalent counterions [30].

It is very instructive to compare our fitting values for $\mu_{D N A}$ and $N_{Z, 0}$ to those obtained for other multivalent counterions. Fitting done for the experiments of DNA condensation with $\mathrm{Spm}^{+4}$ and $\mathrm{Spd}^{+3}$ shows $\mu_{D N A}$ to be -0.07 and $-0.02 k_{B} T$ /base respectively [17, 31]. For our case of $\mathrm{Mg}^{+2}$, a divalent counterion, and bacteriophage $\lambda$ experiment, $\mu_{D N A}$ is found to be $-0.004 k_{B} T /$ base. This is quite reasonable since $\mathrm{Mg}^{+2}$ is a much weaker counterion leading to much lower counterion correlation energy. Furthermore, $N_{Z, 0}$ was found to be 3.2 $\mathrm{mM}$ for the tetravalent counterion, $11 \mathrm{mM}$ for the trivalent counterion. Our fit of $N_{Z, 0}=64$ $\mathrm{mM}$ for divalent counterions again is in favorable agreement with these independent fits. Note that in the limit of high counterion valency $(Z \rightarrow \infty)$, Eq. (9) shows that $N_{Z, 0}$ varies exponentially with $-Z^{3 / 2}$ [12 14]. The large increase in $N_{Z, 0}$ from $3.2 \mathrm{mM}$ for tetravalent counterions to $11 \mathrm{mM}$ for trivalent counterions, and to $64 \mathrm{mM}$ for divalent counterions is not 
surprising.

It is quantitatively significant to point out that our fitted value $\mu_{D N A}=-0.004 k_{B} T$ per base explains why $\mathrm{Mg}^{+2}$ ions cannot condense DNA in free solution. This energy corresponds to an attraction of $-1.18 k_{B} T$ per persistence length. Since the thermal fluctuation energy of a polymer is about $k_{B} T$ per persistence length, this attraction is too weak to overcome thermal fluctuations. It therefore can only partially condense free DNA in solution [18]. Only in the confinement of the viral capsid can this attraction effect appear in the ejection process. It should be mentioned that computer simulations of DNA condensation by idealized divalent counterions [20, 21] show a weak short-range attraction comparable to our $\mu_{D N A}$. The correlation induced DNA-DNA interaction obtained in the simulation of Ref. 20 matches well with our value of $-0.004 k_{B} T$. This suggests that in the presence of divalent counterions, electrostatic interaction are an important (if not dominant) contribution to DNA-DNA short range interactions inside viral capsid.

The phenomenological constants $\mu_{D N A}$ and $N_{Z, 0}$ depend strongly on the strength of the correlations between multivalent counterions on the DNA surface. The stronger the correlations, the greater the DNA-DNA attraction energy $\left|\mu_{D N A}\right|$ and the smaller the concentration $N_{Z, 0}$. In Ref. 10, $\mathrm{MgSO}_{4}$ salt induces a strong inhibition effect. Due to this, $N_{Z, 0}$ for $\mathrm{MgSO}_{4}$ falls within the experimental measured concentration range and we use these data to fit our theory. $\mathrm{MgCl}_{2}$ induces weaker inhibition, thus $N_{Z, 0}$ for $\mathrm{MgCl}_{2}$ is larger and apparently lies at higher value than the measured range. More data at higher $\mathrm{MgCl}_{2}$ concentrations is needed to obtain reliable fitting parameters for this case. In fact, the value $N_{Z, 0} \simeq 104 \mathrm{mM}$ obtained from the computer simulation of Ref. 20 is nearly twice as large as our semi-empirical results. This demonstrates again that this concentration is very sensitive to the exact calculation of the counterion correlation energy $\mu_{\text {cor }}$. The authors of Ref. 10 also used non-ideality and ion specificity as an explanation for these differences. From our point of view, they can lead to the difference in $\mu_{c o r}$, hence in the value $N_{Z, 0}$. In the future, we plan to complimentary our phenomenological theory with a first principle calculation to understand the "microscopic" quantitative differences between $\mathrm{MgSO}_{4}$ and $\mathrm{MgCl}_{2}$ salts.

Lastly, we would like to point out that the fitted value for the equalibrium distance between neighboring DNA in a bundle, $d_{0} \simeq 27.3 \AA$ is well within the range of various known distances from experiments [8, 17]. 


\section{CONCLUSION}

In conclusion, this paper has shown that divalent counterions such as $\mathrm{Mg}^{+2}$ have strong effects on DNA condensation in a confined environment (such as inside bacteriophages capsid) similar to those of counterions with higher valency. We propose that the non-monotonic dependence of the amount of DNA ejected from bacteriophages has the same physical origin as the reentrant condensation phenomenon of DNA molecules by multivalent counterions. Fitting our semi-empirical theory to available experimental data, we obtain the strength of DNA-DNA short range attraction mediated by divalent counterions. The fitted values agree quantitatively and qualitatively with experimental values from other DNA system and computer simulations. This shows that in the problem of viral DNA package where DNA lateral motion is restricted, divalent counterions can plays an important role similar to that of counterions with higher valency. This fact should to be incorporated in any electrostatic theories of bacteriophage packaging. The strength of short-range DNA-DNA attractions mediated by $\mathrm{MgSO}_{4}$ salt is first obtained by the authors. It provides a good starting point for future works with DNA-DNA condensation in the presence of divalent counterions.

\section{ACKNOWLEDGMENTS}

We would like to thank Doctors Shklovskii, Evilevitch, Fang, Gelbart, Podgornik, Naji, Phillips, Rau, and Parsegian for valuable discussions. TTN acknowledges the hospitality of the Fine Theoretical Physics Institute and the Aspen Physics Center where part of this work was done. TTN acknowledges the support of junior faculty from the Georgia Institute of Technology. SL acknowledges financial support from Korean-American Scientists and Engineers Association (Georgia chapter).

\section{REFERENCES}

${ }^{1}$ D. E. Smith, S. J. Trans, S. B. Smith, S. Grimes, D. L. Anderson, and C. Bustamante, Nature 413, 748 (2001).

${ }^{2}$ A. Evilevitch, L. Lavelle, C. M. Knobler, E. Raspaud, and W. M. Gelbart, Proc. Nat. Acad. Sci. USA 100, 9292 (2003). 
${ }^{3}$ M. Castelnovo, R. K. Bowles, H. Reiss, and W. M. Gelbart, Eur. Phys. J. E 10, 191 (2003).

${ }^{4}$ A. S. Petrov, K. Lim-Hing, and S. C. Harvey, Structure 15, 807 (2007).

${ }^{5}$ L. Letellier, P. Boulanger, L. Plancon, P. Jacquot, and M. Santamaria, Front. Biosci. 9, $1228(2004)$.

${ }^{6}$ L. W. Black, Annu. Rev. Microbiol. 43, 267 (1989).

${ }^{7}$ H. Murialdo, Annu. Rev. Biochem. 60, 125 (1991).

${ }^{8}$ P. K. Purohit, M. M. Inamdar, P. D. Grayson, T. M. Squires, J. Kondev, and R. Phillips, Biophys. J. 88, 851 (2005).

${ }^{9}$ A. Evilevitch, M. Castelnovo, C. M. Knobler, and W. M. Gelbart, J. Phys. Chem. B 108, 6838 (2004).

${ }^{10}$ A. Evilevitch, L. T. Fang, A. M. Yoffe, M. Castelnovo, D. C. Rau, V. A. Parsegian, W. M. Gelbart, and C. M. Knobler, Biophys. J. 94, 1110 (2008).

${ }^{11}$ L. T. Fang, private communications (2009).

${ }^{12}$ B. I. Shklovskii, Phys. Rev. E 60, 5802 (1999).

${ }^{13}$ A. Y. Grosberg, T. T. Nguyen, and B. Shklovskii, Rev. Mod. Phys. 74, 329 (2002).

${ }^{14}$ A. G. Moreira and R. R. Netz, Eur. Phys. J. E 8, 33 (2002).

${ }^{15}$ K. Besteman, K. V. Eijk, and S. G. Lemay, Nature Physics 3, 641 (2007).

${ }^{16}$ M. Kanduč, A. Naji, and R. Podgornik, J. Chem. Phys. 132, 224703 (2010).

${ }^{17}$ D. C. Rau and V. A. Parsegian, Biophys. J. 61, 246 (1992).

${ }^{18}$ N. V. Hud and K. H. Downing, Proc. Nat. Acad. Sci. USA 98, 14925 (2001).

${ }^{19}$ I. Koltover, K. Wagner, and C. R. Safinya, Proc. Nat. Acad. Sci. USA 97, 14046 (2000).

${ }^{20}$ S. Lee, T. T. Le, and T. T. Nguyen, Phys. Rev. Lett. (Accepted for publication) (2010), arXiv:cond-mat/0912.3595.

${ }^{21}$ A. P. Lyubartsev and L. Nordenskiöld, J. Phys. Chem. 99, 10373 (1995).

${ }^{22}$ M. Winterhalter and W. Helfrich, J. Phys. Chem. 92, 6865 (1988).

${ }^{23}$ V. I. Perel and B. I. Shklovskii, Physica A 274, 446 (1999).

${ }^{24}$ R. Bruinsma, Eur. Phys. J. B 4, 75 (1998).

${ }^{25}$ W. M. Gelbart, R. F. Bruinsma, P. A. Pincus, and A. V. Parsegian, Phys. Today 53, 38 (2000).

${ }^{26}$ S. C. Riemer and V. A. Bloomfield, Biopolymers 17, 785 (1978). 
${ }^{27}$ J. Kindt, S. Tzlil, A. Ben-Shaul, and W. M. Gelbart, Proc. Nat. Acad. Sci. USA 98, 13671 (2001).

${ }^{28}$ A. S. Petrov and S. C. Harvey, Biophys. J. 95, 497 (2008).

${ }^{29}$ T. S. Baker, N. H. Olson, and S. D. Fuller, Microbiol. Mol. Biol. Rev. 63, 862 (1999).

${ }^{30}$ B. Skinner and B. I. Shklovskii, Physica A 388, 1 (2009).

${ }^{31}$ T. T. Nguyen, I. Rouzina, and B. I. Shklovskii, J. Chem. Phys. 112, 2562 (2000). 